\title{
RANKED FRAGMENTATIONS
}

\author{
JULIEN BERESTYCKI ${ }^{1}$
}

\begin{abstract}
In this paper we define and study self-similar ranked fragmentations. We first show that any ranked fragmentation is the image of some partition-valued fragmentation, and that there is in fact a one-to-one correspondence between the laws of these two types of fragmentations. We then give an explicit construction of homogeneous ranked fragmentations in terms of Poisson point processes. Finally we use this construction and classical results on records of Poisson point processes to study the small-time behavior of a ranked fragmentation.
\end{abstract}

Mathematics Subject Classification. 60J25, 60G09.

Received October 30, 2001. Revised March 28, 2002.

\section{INTRODUCTION}

Splitting models are meant to describe an object that falls apart. Applications are numerous and may be found in various fields such as physical chemistry (aerosols, phase separation, polymerization), mathematical population genetics or astronomy (we refer to [2] for a survey on applications and motivations).

This paper focuses on self-similar ranked fragmentations. For the sake of describing our results, let us just give some heuristic descriptions while precise definitions will be given in the next sections.

Imagine a unit-mass object that fragments as time runs. We only consider the ordered sequence of the masses of the fragments so the state space is

$$
\mathcal{S}^{\downarrow}:=\left\{s=\left(s_{1}, s_{2}, \ldots\right), s_{1} \geq s_{2} \geq \ldots \geq 0, \sum_{i} s_{i} \leq 1\right\},
$$

the situation where $\sum_{i} s_{i}<1$ corresponding to the fact that a part of the initial mass has been lost, i.e. the sum of the masses of the remaining fragments is less than the original total mass.

Let $\lambda=(\lambda(t), t \geq 0)$ be a Markov process with values in $\mathcal{S}^{\downarrow}$. Call $\lambda$ a self-similar ranked fragmentation if it fulfills the scaling and fragmentation properties.

The scaling property means that there exists a real number $\alpha$, called the index of self-similarity, such that if $\mathbb{P}_{r}$ is the law of $\lambda$ started from $(r, 0,0, \ldots)$ then the distribution of $\left(r \lambda\left(r^{\alpha} t\right), t \geq 0\right)$ under $\mathbb{P}_{1}$ is $\mathbb{P}_{r}$.

The fragmentation property is a version of the branching property i.e. for any $u, t \geq 0$, for any $s=$ $\left(s_{1}, s_{2}, \ldots\right) \in \mathcal{S}^{\downarrow}$, conditionally on $\lambda(u)=s, \lambda(t+u)$ has the same distribution as the variable obtained by concatenating and ordering the sequences $\lambda^{(1)}, \lambda^{(2)}, \ldots$ where for each $i, \lambda^{(i)}$ has the distribution of $\lambda(t)$ under $\mathbb{P}_{s_{i}}$.

Keywords and phrases: Fragmentation, self-similar, subordinator, exchangeable partitions, record process.

${ }^{1}$ Laboratoire de Probabilités et Modèles Aléatoires, Université Pierre et Marie Curie, UMR 7599 du CNRS,

175 rue du Chevaleret, 75013 Paris, France; e-mail: jberest@ccr.jussieu.fr 
Here is a simple prototype taken from Brennan and Durrett $[10,11]$ who consider the following model for polymer degradation: A particle of mass $m$ splits with exponential rate $m^{\alpha}, \alpha \in \mathbb{R}^{+}$, and gives rise to two particles of mass $V m$ and $(1-V) m$, where $V$ is a random variable with values in $(0,1)$ independent of the past. The new particles follow the same dynamic independently. The ordered sequence of the masses of the particles is a self-similar ranked fragmentation of index $\alpha$.

This example can be extended in two ways. First one can suppose that when a particle splits, it might give birth to any number of particles, possibly infinite, and not just two. Second, in the example of Brennan and Durrett, the splitting times are "discrete", the first time of splitting is almost surely strictly positive. It is natural to consider more generally the case where fragmentation may occur continuously. For instance this happens for the fragmentation process obtained by logging the continuous random tree of Aldous in [3].

A classical tool in the study of ranked fragmentations or coalescences is to use a somewhat different statespace and to introduce the so-called partition valued fragmentation (see for instance Kingman in [15] or Evans and Pitman in [13]). Roughly speaking a partition fragmentation, say $\Pi(t)$, is a process that lives in the space of partitions of $\mathbb{N}$, such that for any $0<s \leq t, \Pi(t)$ is a refinement of $\Pi(s)$. A way to construct such a fragmentation which makes clear the connection with the above particle model is the following: imagine an object $E$ endowed with a unit mass measure $\mu$ that falls apart as time runs. Call object fragmentation the process $F(t)$ with values in partitions of $E$ that describes this fragmentation. Next, let $\left(u_{i}\right)_{i \in \mathbb{N}}$ be a sequence of iid $E$-valued variables with distribution $\mu$ and for each $t$ let $\Pi_{F}(t)$ be the partition of $\mathbb{N}$ such that for all $i$ and $j$ in $\mathbb{N}, i$ and $j$ belong to the same block of $\Pi(t)$ iff $u_{i}$ and $u_{j}$ are in the same fragment of $E$ at time $t$. By the SLLN we can recover the mass of a fragment as the asymptotic frequency of the corresponding block. Then $\Pi_{F}$ is a partition fragmentation.

Using partition fragmentations to construct ranked fragmentations is typical of the existing results. These constructions benefit from two important features: there is a clear genealogical structure, and partition fragmentations, when they are self-similar, are characterized by an index of self-similarity and a so-called characteristic exchangeable measure, on which results concerning exchangeability can be usefully applied (see [1] for a survey on exchangeability).

However partition-valued fragmentations are perhaps less natural and could be less general than ranked fragmentations, precisely because we have endowed them with this extra genealogic structure. In other words it is not clear that an arbitrary ranked fragmentation can be studied through partition fragmentations.

In Section 2 we show that it is in fact the case. More precisely, if we give ourselves a ranked fragmentation $\lambda$, there is a partition fragmentation $\Pi$ such that the asymptotic frequencies of $\Pi$ have the distribution as $\lambda$.

In the next section we use this equivalence between ranked and partition fragmentations to give a Poisson construction of homogeneous ranked fragmentation which is an analogue of that given in [5] for partition fragmentations. The difficulty comes from the fact that we can no longer use a genealogic structure, which played a crucial role in the partition case.

In Section 4, this construction allows us to tackle the study of small time behavior of a ranked fragmentation. We show that the $2^{\text {nd }}$ largest fragment, correctly renormalized, behaves as the record of the size of the particles detaching from the main fragment.

\section{Definitions AND FIRST PROPERTIES}

\subsection{Ranked fragmentations}

For each $l$ in $[0,1]$ let $P(l)$ be a probability on $\left\{s \in \mathcal{S}^{\downarrow}: \sum_{i} s_{i} \leq l\right\}$, the space of all the possible fragmentations of $l$. Then for $L=\left(l_{1}, l_{2}, \ldots\right) \in \mathcal{S}^{\downarrow}$, define $P(L)$ as the distribution on $\mathcal{S}^{\downarrow}$ of the concatenation and the decreasing rearrangement of independent $\mathcal{S}^{\downarrow}$-valued variables with respective law $P\left(l_{i}\right)$. Call $\left(P(L), L \in \mathcal{S}^{\downarrow}\right)$ a fragmentation kernel on $\mathcal{S}^{\downarrow}$. One says that the family $(P(l), l \in[0,1])$ generates $\left(P(L), L \in \mathcal{S}^{\downarrow}\right)$. 
Definition 2.1. An $\mathcal{S}^{\downarrow}$-valued process $\lambda\left(\right.$.) is called a $\mathcal{S}^{\downarrow}$-fragmentation if it is a time-homogeneous Markov process such that

1. $\lambda$ is continuous in probability and starts from $\lambda(0)=(1,0,0, \ldots)$ a.s.;

2. the transition semigroup $\left(P_{t}(L)\right)$ of $\lambda$ is given by fragmentation kernels.

In words, at a given time $t$, each fragment of $\lambda(t)=\left(\lambda_{1}(t), \lambda_{2}(t), \ldots\right)$, say $\lambda_{i}(t)$, gives rise to an independent fragmentation process whose distribution only depends on the value $\lambda_{i}(t) . \quad \lambda$ is the concatenation and the reordering of all those processes.

For $l \in[0,1]$, let $g_{l}$ be the function from $\mathcal{S}^{\downarrow} \rightarrow \mathcal{S} \downarrow$ defined by

$$
g_{l}: x=\left(x_{1}, x_{2}, \ldots\right) \rightarrow\left(l x_{1}, l x_{2}, \ldots\right) .
$$

Definition 2.2. The fragmentation $\lambda$, with transition kernels generated by the family $\left(P_{t}(l) ; t \geq 0, l \in[0,1]\right)$ is said to be self-similar with index $\alpha \in \mathbb{R}$ if (in the notations introduced above) for all $l \in[0,1]$ the distribution $P_{t}(l)$ coincides with the image of $P_{l^{\alpha}}(1)$ by $g_{l}$.

When $\alpha=0$ the fragmentation is said to be homogeneous.

$\mathcal{S} \downarrow$ is endowed with the uniform distance. Note that for any $s=\left(s_{1}, s_{2}, \ldots\right) \in \mathcal{S}^{\downarrow}$ we must have for every $k \in \mathbb{N}, s_{k} \leq \frac{1}{k}$, and thus the uniform and pointwise convergences are the same.

In [5] Bertoin showed that the semi-group of a homogeneous partition fragmentation (the definition and main properties of partition fragmentations are discussed in Sect. 2.2) has the Feller property. As we are working with a different topology his result does not apply. However, we have the following:

Proposition 2.3. (Feller property) Under the weak topology on probability measures, the semi-group $P_{t}$ of a self-similar ranked fragmentation of index $\alpha$, fulfills the Feller property. That is $\forall t \geq 0$ the map

$$
L \rightarrow P_{t}(L)
$$

is continuous on $\mathcal{S}^{\downarrow}$ and for each fixed $L \in \mathcal{S}^{\downarrow}, P_{t}(L)$ converges to the Dirac mass at $L$ as $t \rightarrow 0$.

Proof. The second point is simply the continuity in probability of a self-similar fragmentation.

For the first point consider a sequence $\left(L_{n}, n \in \mathbb{N}\right)$ in $\mathcal{S}^{\downarrow}$ which converges to $L \in \mathcal{S}^{\downarrow}$. Note $L_{n}=\left(l_{1}^{(n)}, l_{2}^{(n)}, \ldots\right)$, then for all $k, l_{k}^{(n)} \rightarrow l_{k}$.

Let $\left(Y_{i}(t)\right)_{i \in \mathbb{N}}$ be a sequence of iid $\mathcal{S}^{\downarrow}$-fragmentations with same semi-group $\left(P_{t}(S), S \in \mathcal{S}^{\downarrow}, t \geq 0\right.$ ), then, by definition, for all $n \in \mathbb{N}$ the $\mathcal{S}^{\downarrow}$ random variable $Z^{(n)}(t)$, obtained by the decreasing rearrangement of the terms $g_{l_{i}^{(n)}}\left(Y_{1}\left(t\left(l_{i}^{(n)}\right)^{\alpha}\right)\right)$ for $i$ in $\mathbb{N}$ :

$$
Z^{(n)}(t)=\left(g_{l_{1}^{(n)}}\left(Y_{1}\left(t\left(l_{1}^{(n)}\right)^{\alpha}\right)\right), g_{l_{2}^{(n)}}\left(Y_{2}\left(t\left(l_{2}^{(n)}\right)^{\alpha}\right)\right), \ldots\right)^{\downarrow}
$$

has law $P_{L^{(n)}}(t)$. In the same way

$$
Z(t)=\left(g_{l_{1}}\left(Y_{1}\left(t\left(l_{1}\right)^{\alpha}\right)\right), g_{l_{2}}\left(Y_{2}\left(t\left(l_{2}\right)^{\alpha}\right)\right), \ldots\right)^{\downarrow}
$$

has law $P_{L}(t)$. Now fix $\epsilon>0$, and take $N>\frac{2}{\epsilon}$. Then

$$
\forall k \geq N, \forall n \in \mathbb{N}, l_{k}^{(n)}<\epsilon / 2 .
$$

Thus for all $\omega$

$$
\sup _{k \geq N}\left(\operatorname{dist}\left(g_{l_{k}^{(n)}}\left(Y_{k}\left(t\left(l_{k}^{(n)}\right)^{\alpha}\right)\right), g_{l_{k}}\left(Y_{k}\left(t\left(l_{k}\right)^{\alpha}\right)\right)\right)\right)<\epsilon .
$$


On the other hand, by the continuity in probability of the proceeses $\left(Y_{i}\right)_{i \in\{1, \ldots, N-1\}}$, we have that almost surely

$$
P\left[\sup _{k \in\{1, \ldots, N-1\}}\left(\operatorname{dist}\left(g_{l_{k}^{(n)}}\left(Y_{k}\left(t\left(l_{k}^{(n)}\right)^{\alpha}\right)\right), g_{l_{k}}\left(Y_{k}\left(t\left(l_{k}\right)^{\alpha}\right)\right)\right)\right)>\epsilon\right] \underset{n \rightarrow \infty}{\rightarrow} 0 .
$$

Thus almost surely, for all $\epsilon>0$, there exists $N \in \mathbb{N}$ such that for all $n \geq N$

$$
\operatorname{dist}\left(Z^{(n)}(t), Z(t)\right)<\epsilon .
$$

There is convergence in probability and thus in law.

\subsection{Partition fragmentations}

Most of the results on fragmentation available in the literature are (or can be) formulated in terms of a type of fragmentation called partition fragmentation, which is basically a process that can be described as a partition of $\mathbb{N}$ getting finer as time runs. We recall in this section several well-known facts that we will use repeatedly in the following. We refer to Bertoin [5], Bolthausen and Sznitman [9], Evans and Pitman [13] and Kingman [15] (among others) for results and discussions on partition valued processes and the link between partition fragmentations/coalescences and ranked fragmentations/coalescences.

More precisely, call a subset of $\mathbb{N}$, say $B$, a "block". When the limit

$$
|B|:=\lim _{n \rightarrow \infty} \frac{1}{n} \operatorname{Card}\{0 \leq k \leq n: k \in B\}
$$

exists, it is called the asymptotic frequency of $B$. A partition of $\mathbb{N}$ can be thought of as a sequence $B_{1}, B_{2}, \ldots$ of disjoint blocks whose union is $\mathbb{N}$. The labeling obeys the following rule: if $B_{i}$ is not empty, then its least element is $i$. Call $\mathcal{P}$ the space of the partitions of $\mathbb{N}$, and recall that $\mathcal{P}$ is a compact metric space (see [15]).

A finite permutation $\sigma$ (i.e. a bijection $\mathbb{N} \rightarrow \mathbb{N}$ such that $\sigma(n)=n$ for $n$ large enough) acts on a partition $\pi$ in the following way: for any $i$ and $j$ in $\mathbb{N}, i$ and $j$ are in the same block of $\sigma(\pi)$ iff $\sigma(i)$ and $\sigma(j)$ are in the same block of $\pi$, this equivalence relation can be identified as a partition and thus completely defines $\sigma(\pi)$.

A measure $\mu$ on $\mathcal{P}$ is said to be exchangeable if for any measurable set $A \subseteq \mathcal{P}$, for any finite permutation $\sigma$

$$
\mu(A)=\mu(\sigma(A))
$$

where $\sigma$ acts on the sets in the obvious way.

A $\mathcal{P}$-valued process $\Pi$ is said to be exchangeable if the permuted process $\sigma(\Pi)$ has the same distribution as the original process $\Pi$. For instance the $\mathcal{P}$-valued process $\Pi_{F}(t)$ presented in the introduction is exchangeable.

For all $B \subseteq \mathbb{N}$, let $P_{B}$ be a probability on the partitions of $B$. For all $\pi=\left(B_{1}, B_{2}, \ldots\right) \in \mathcal{P}$, let $P_{\pi}$ be the distribution of the partition with blocks $B_{(1,1)}, B_{(1,2)}, \ldots, B_{(2,1)}, B_{(2,2)}, \ldots$ where $\pi^{(i)}=\left(B_{(i, 1)}, B_{(i, 2)}, \ldots\right)$ is a partition of $B_{i}$ and has law $P_{B_{i}}$. The family $\left(P_{\pi}, \pi \in \mathcal{P}\right)$ is, in the terminology of Pitman [17], a fragmentation kernel on $\mathcal{P}$.

Definition 2.4. Call $\mathcal{P}$-fragmentation any exchangeable $\mathcal{P}$-valued Markov process, starting from the trivial partition ( $\mathbb{N}$ is the only non empty block), which is continuous in probability and has fragmentation kernels as its transition semi-group.

If $\pi$ is a random exchangeable partition, by a result of Kingman [15] (see also Aldous [1] for a simpler proof), every block of $\pi$ has an asymptotic frequency almost surely, i.e. $\left|B_{i}\right|$ exists with probability 1 for all $i=1, \ldots$

We call an exchangeable $\mathcal{P}$-valued process $\Pi$ nice if with probability $1, \Pi(t)$ has asymptotic frequencies for all $t \geq 0$ simultaneously. Evans and Pitman [13] have shown that it is always the case when $\Pi$ is an exchangeable $\mathcal{P}$-process with proper frequencies (i.e. for each $t \geq 0, \sum_{i \in \mathbb{N}}\left|B_{i}(t)\right|=1$ almost surely), and Bertoin [5] proved 
that so-called homogeneous fragmentation were nice. Observe that when $\Pi(t)$ is nice, the ordered sequence of the asymptotic frequencies is well defined and is a $\mathcal{S} \downarrow$-valued process.

As we shall construct a Markovian semi-group on $\mathcal{S}^{\downarrow}$, we need a notion slightly more general than the asymptotic frequency, well defined for any subset $B$ of $\mathbb{N}$. We write

$$
\Lambda(\Pi(t))=\left(\Lambda_{1}(\Pi(t)), \Lambda_{2}(\Pi(t)), \ldots\right)^{\downarrow}=\left(\lambda_{1}(t), \lambda_{2}(t), \ldots\right)
$$

for the decreasing rearrangement of the quantities

$$
\Lambda_{i}(\Pi(t))=\liminf _{n \rightarrow \infty} \frac{1}{n} \#\left\{k \leq n: k \in B_{i}(t)\right\} .
$$

By extension we also define

for any $B \subseteq \mathbb{N}$.

$$
\Lambda(B)=\liminf _{n \rightarrow \infty} \frac{1}{n} \#\{k \leq n: k \in B\}
$$

$\Lambda$ is a functional of $\Pi(t)$ that takes its values in $\mathcal{S}^{\downarrow}$. We stress that $\Lambda$ is not continuous.

Next for every $C \subseteq \mathbb{N}$ and every $\pi=\left\{B_{1}, B_{2}, \ldots\right\} \in \mathcal{P}$, we define the partition of $C$ induced ${ }^{2}$ by $\pi$ :

$$
\pi \cap C=\left(B_{1} \cap C, B_{2} \cap C, \ldots\right) .
$$

Definition 2.5. A $\mathcal{P}$-fragmentation $\Pi=(\Pi(t), t \geq 0)$ is called self-similar with index $\alpha \in \mathbb{R}$ if:

1. $\Pi$ starts a.s. from the trivial partition;

2. the ranked fragmentation $\Lambda(\Pi)$ associated with $\Pi$ is continuous in probability;

3. for every $B \subseteq \mathbb{N}, \forall t \geq 0 P_{B}(t)$ (in the above notations) is the distribution of $\Pi\left(t \Lambda(B)^{\alpha}\right) \cap B$.

When $\alpha=0$ we will say that $\Pi$ is a homogeneous fragmentation.

Following Kingman [15] (see also [1] for a survey), to each $s=\left(s_{1}, s_{2}, \ldots\right) \in \mathcal{S}^{\downarrow}$ one can associate a unique exchangeable probability measure $\mu_{s}$ on $\mathcal{P}$ such that $\mu_{s}$-almost every partition has ranked asymptotic frequencies $s$.

This is how one proceeds: let $\left(X_{i}\right)_{i \in \mathbb{N}}$ a family of iid variables such that $\forall k \in \mathbb{N}, P\left(X_{i}=k\right)=s_{k}$ and $P\left(X_{i}=0\right)=1-\sum_{k} s_{k}$, then define the $s$-paintbox ${ }^{3}$ partition (or " $s$-paintbox process") $\Pi$ by the equivalence relation

$$
\forall i, j \in \mathbb{N}, i \sim j \Leftrightarrow X_{i}=X_{j}>0
$$

We denote by $\mu_{s}$ the law of the $s$-paintbox process. It is clear by the LLN that $\mu_{s}$-almost surely $\Lambda(\Pi)=s$.

For each self-similar $\mathcal{P}$-fragmentation one can take the associated $\mathcal{S} \downarrow$ ranked fragmentation, thus defining a map from $\mathcal{P}$-fragmentation laws into $\mathcal{S} \downarrow$-fragmentation laws. Suppose now that $\Pi_{1}$ and $\Pi_{2}$ are two selfsimilar $\mathcal{P}$-fragmentations such that for any fixed $t$ the $\mathcal{S} \downarrow$ variables $\Lambda\left(\Pi_{1}(t)\right)$ and $\Lambda\left(\Pi_{2}(t)\right)$ have same law. $\Pi_{1}(t)$ and $\Pi_{2}(t)$ being exchangeable, by de Finetti's theorem (see [1]) one can show that they are mixtures of paintbox processes directed respectively by $\Lambda\left(\Pi_{1}\right)$ and $\Lambda\left(\Pi_{2}\right)$, i.e.

$$
P\left(\Pi_{1,2} \in A\right)=\int_{\mathcal{S} \downarrow} \mu_{s}(A) P\left(\Lambda\left(\Pi_{1,2}\right) \in \mathrm{d} s\right) .
$$

\footnotetext{
${ }^{2}$ There is in fact another natural way of defining this partition: it is to take the image of $\pi$ by the mapping that sends $\mathbb{N}$ onto $C=\left\{c_{1}, c_{2}, \ldots\right\}$ (where $\left.c_{1}<c_{2}<\ldots\right)$ :

$$
\pi \circ C=\left(\left\{c_{j}: j \in B_{i}\right\}_{i=1, \ldots}\right) .
$$

Suppose now that $\pi$ is an exchangeable random $\mathcal{P}$-valued variable, for all $k>0$, for any finite permutation $\sigma$ such that

$$
\forall i \leq k ; \sigma(i)=c_{i},
$$

$\pi$ and $\sigma(\pi)$ have same law, thus in the sense of the equality of the finite-dimensional margins $\pi \circ C$ and $\pi \cap C$ have same law.

${ }^{3}$ The reason for the name (due to Kingman) is the following: imagine that we have a choice of colors $\left(c_{k}\right)_{k \in \mathbb{N}}$. Then paint each integer $n$ independently with a randomly chosen color, $c_{k}$ with probability $s_{k}$. Then the partition of $\mathbb{N}$ defined by the equivalence relation "being of the same color" is the $s$-paintbox process.
} 
We conclude that they have the same distribution. So to every $\mathcal{P}$-fragmentation corresponds a different $\mathcal{S} \downarrow$-fragmentation. Our first result will be to show that there is in fact a one to one relation.

\subsection{From ranked to partition fragmentations}

Let $\Pi$ be a nice self-similar fragmentation of index $\alpha$, then it is known (see $[5,15]$ ) that its asymptotic frequencies $\Lambda(\Pi)$ form a self-similar ranked fragmentation of index $\alpha$. In the converse direction, Evans and Pitman in [13] showed that given a ranked fragmentation that admits a so-called tracking function one can always see it as the image of a corresponding partition fragmentation. We shall extend this result by showing that this is the case for any $\mathcal{S}^{\downarrow}$-fragmentation, which means that any self-similar $\mathcal{S}^{\downarrow}$-fragmentation admits a family of tracking functions.

Proposition 2.6. We have the following relations between $\mathcal{S} \downarrow$ and $\mathcal{P}$ fragmentations:

1. if $\Pi$ is a $\mathcal{P}$-fragmentation then $\Lambda(\Pi)$ has the finite-dimensional marginal distributions of an $\mathcal{S} \downarrow$-fragmentation. Moreover $\Lambda$ preserves self-similarity;

2. if $\lambda$ is a $\mathcal{S}^{\downarrow}$-fragmentation, then we can construct $\Pi_{\lambda}$ an exchangeable $\mathcal{P}$-fragmentation such that $\Lambda\left(\Pi_{\lambda}\right) \stackrel{\mathcal{L}}{=}$ $\lambda$. Moreover this construction preserves self-similarity.

The first point is clear, the difficulty here lies in the second part of this proposition. The main idea is that as $\mathcal{P}$ is a compact metric space, it is enough to construct an adequate Markovian semi-group to ensure the existence of the desired $\mathcal{P}$-process. Then the conservation of the index will be a simple consequence of our construction.

Let $\left(P_{t}(S), t \geq 0, S \in \mathcal{S}^{\downarrow}\right)$ be a transition kernel on $\mathcal{S}^{\downarrow}$ generated, in the notation of Definition 2.1, by the family $\left(P_{t}(l), t \geq 0, l \in[0,1]\right)$.

Let $\tilde{P}_{t}(l)$ be the image of $P_{t}(l)$ by $g_{l^{-1}}$, the map $\left(x_{1}, x_{2}, \ldots\right) \rightarrow\left(x_{1} / l, x_{2} / l, \ldots\right)$. Let $(R(t, l), l \in[0,1], t \geq 0)$ be a family of probability measures on $\mathcal{P}$ where, for a fixed $t, R(t, l)$ is a mixture of $s$-paintbox processes directed by $\tilde{P}_{t}(l)$, i.e. for $A \subseteq \mathcal{P}$

$$
R(t, l)(A)=\int_{\mathcal{S} \downarrow} \mu_{s}(A) \tilde{P}_{t}(l, \mathrm{~d} s) .
$$

For $B \subseteq \mathbb{N}$ define $Q(t, B)$ the distribution of $\Pi_{B} \cap B$ where $\Pi_{B}$ is a $\mathcal{P}$ valued random variable with law $R(t, \Lambda(\bar{B}))$. Practically this means that one begins by drawing a variable $\lambda_{B}$ with law $\tilde{P}_{t}(\Lambda(B))$, we then take the sub-partition of $B$ induced by the $\lambda_{B}$-paintbox partition.

Now let $\pi=\left(\pi_{1}, \pi_{2}, \ldots\right) \in \mathcal{P}$ and $\forall t \geq 0$ let $\left(\Pi_{\pi_{i}}(t)\right)_{i \in \mathbb{N}}$ be a sequence of independent variables with respective law $Q\left(t, \pi_{i}\right)$. Define $Q(t, \pi)$ the law of the partition whose blocks are the blocks of the $\left(\Pi_{\pi_{i}}(t), i \in \mathbb{N}\right)$.

Our proof of Proposition 2.6 shall thus consist of showing that the family $(Q(t, \pi), \pi \in \mathcal{P}, t \geq 0)$ forms a semi-group.

Proof. From the above description it should be clear that it suffices to show

$$
\forall \pi \in \mathcal{P}, Q(t+u, \pi)=\int_{\pi^{\prime} \in \mathcal{P}} Q\left(t, \pi^{\prime}\right) Q(u, \pi)\left(\mathrm{d} \pi^{\prime}\right)
$$

in the obvious notation. If for any subset $B$ of $\mathbb{N}$, we denote the set of partitions of $B$ by $\mathcal{P}_{B}$, then the construction, (1) is equivalent to

$$
\forall B \subseteq \mathbb{N}, Q(t+u, B)=\int_{\pi^{\prime} \in \mathcal{P}_{B}} Q\left(t, \pi^{\prime}\right) Q(u, B)\left(\mathrm{d} \pi^{\prime}\right)
$$


We can reformulate $(2)$ as: $Q(t+u, B)$ is the distribution of the random partition $\Pi(t, u)$ of $B$ (and this is what we actually shall prove) obtained by the following two-step procedure:

1. draw $\Pi(u)=\left(\pi_{1}(u), \pi_{2}(u), \ldots\right)$ an exchangeable partition of $B$ with law $Q(u, B)$;

2. given $\Pi(u)$ draw a sequence $\left(\Pi_{\pi_{i}(u)}(t)\right)_{i \in \mathbb{N}}$ of independent $\mathcal{P}_{\pi_{i}(u)}$-variables with respective law $Q\left(t, \pi_{i}(u)\right)_{i \in \mathbb{N}}$;

3. $\Pi(t, u)$ is just the collection of all the blocks of the $\Pi_{\pi_{i}(u)}(t)$.

We begin by proving so for $B=\mathbb{N}$. By construction we can always suppose that $\Pi(u)$ is a mixture of paintbox processes directed by $P_{u}\left((1,0, \ldots)\right.$ ), i.e. conditionally on $\lambda(u)$ (a random variable with law $P_{u}((1,0, \ldots))$ ), $(u)$ is a $\lambda(u)$-paintbox process (resp. for each $i \in \mathbb{N}, \Pi_{\pi_{i}(u)}(t)$ is constructed by taking the intersection of $\pi_{i}(u)$ and a $\lambda^{(i)}$ paintbox-process where $\lambda^{(i)}$ is a $\mathcal{S}^{\downarrow}$-variable with law $\left.\tilde{P}_{t}\left(\left|\pi_{i}(u)\right|\right)\right)$.

This means that conditionally on $\lambda(u)=\left(\lambda_{1}(u), \lambda_{2}(u), \ldots\right)$, one draws an i.i.d. sequence of random variables $\left(X_{i}\right)_{i \in \mathbb{N}}$ with values in $\{0,1,2, \ldots\}$ such that $P\left(X_{1}=k\right)=\lambda_{k}(u)$ for all $k \geq 1$ and $P\left(X_{i}=0\right)=1-\sum_{n} \lambda_{n}(u)$. These random variables determine $\Pi(u)$. in the same way, for each $i \in \mathbb{N}$, conditionally on $\lambda^{(i)}$, one draws an i.i.d. sequence of random variable $\left(Y_{k}^{(i)}\right)_{k \in \mathbb{N}}$ which determines $\Pi_{\pi_{i}(u)}(t)$. Note that $i \stackrel{\Pi(t, u)}{\sim} j$ if and only if $X_{i}=X_{j}>0$ and $Y_{i}^{\left(X_{i}\right)}=Y_{j}^{\left(X_{j}\right)}>0$. Since $\left(X_{i}\right)_{i \in \mathbb{N}}$ and $\left(Y_{k}^{(i)}\right)_{k \in \mathbb{N}}$ are i.i.d. sequences, it follows that $\Pi(t, u)$ is exchangeable.

The law of an exchangeable random partition is completely determined by the law of its asymptotic frequencies, here the $\lambda_{i}(u) \times \lambda^{(i)}(t)$. As $\lambda($.$) is a \mathcal{S}^{\downarrow}$-fragmentation we have by construction that

$$
\left(\left(\lambda_{i}(u) \times \lambda^{(i)}(t)\right)_{i \in \mathbb{N}}\right)^{\downarrow} \stackrel{\mathcal{L}}{=} \lambda(t+u)
$$

So $\Pi(t, u)$ has law $Q(t+u,\{\mathbb{N}\})$.

Then take $B$ a subset of $\mathbb{N}$. By construction $Q(t+u, B)$ is the law of $\tilde{\Pi}_{t+u}(B) \cap B$ where $\tilde{\Pi}_{t+u}(B)$ is a $\mathcal{P}$-variable and $\Pi(t, u)=\tilde{\Pi}(t, u) \cap B$ where $\tilde{\Pi}(t, u)$ is a $\mathcal{P}$-variable. It is clear that replacing the generating family $(R(t, l), t \geq 0, l \in[0,1])$ by $\left(R^{\prime}(t, l)=R(t, \Lambda(B) l), t \geq 0, l \in[0,1]\right)$ in the above arguments yields $\tilde{\Pi}(t, u) \stackrel{\mathcal{L}}{=} \tilde{\Pi}_{t+u}(B)$ and thus for all $B \subseteq \mathbb{N}$

$$
\Pi(t, u) \stackrel{\mathcal{L}}{=} \Pi_{t+u}(B)
$$

So we have proved the existence of a Markov $\mathcal{P}$-process $\Pi$ with semi-group $Q(t, \pi)$, which, by construction, is a fragmentation whose asymptotic frequencies have same distribution (in the sense of finite-dimensional distributions) as $\lambda_{t}$ our starting $\mathcal{S}^{\downarrow}$-fragmentation.

For each ranked fragmentation $\lambda$ we can thus construct a partition fragmentation $\Pi_{\lambda}$ such that $\Lambda\left(\Pi_{\lambda}\right)$ has same law as $\lambda$.

We now turn to the conservation of self-similarity: suppose $\lambda$ is a self-similar $\mathcal{S} \downarrow$-fragmentation with index $\alpha$, so $\tilde{P}_{l}(t)=P_{1}\left(t l^{\alpha}\right)$, looking at the above construction of the semi-group shows that $R(t, l)=R\left(t l^{\alpha}, 1\right)$, so $\Pi$ is also self-similar of index $\alpha$.

It is now natural to look for some explicit construction of ranked fragmentations, i.e. an equivalent of Theorem 1 in [5].

\section{Homogeneous fragmentations}

In [5] J. Bertoin shows how a homogeneous $\mathcal{P}$-fragmentation process can be decomposed into a Poisson point process of partitions, whose distribution is determined by the so-called characteristic measure. As one can always suppose that a $\mathcal{S}^{\downarrow}$-fragmentation is the image by $\Lambda$ of some $\mathcal{P}$-fragmentation, one might ask if it is possible to construct an $\mathcal{S}^{\downarrow}$-fragmentation directly from some Poisson point process. 


\subsection{Lévy-Itô decomposition of homogeneous $\mathcal{P}$-fragmentations}

We start by recalling some results lifted from [5]. The distribution of a homogeneous $\mathcal{P}$-fragmentation $\Pi$ is determined by an exchangeable measure $\kappa$ on $\mathcal{P}$, called the characteristic measure of $\Pi$, that assigns zero mass to the trivial partition and satisfies the condition $\kappa\left(\mathcal{P}_{2}^{*}\right)<\infty$ where $\mathcal{P}_{2}^{*}$ is the set of the partitions of $\mathbb{N}$ for which 1 and 2 do not belong to the same block. Given such a measure $\kappa$, one can construct a homogeneous $\mathcal{P}$-fragmentation admitting $\kappa$ as its characteristic measure as follows: let $K=((\Delta(t), k(t)), t \geq 0)$ a Poisson point process with values in $\mathcal{P} \times \mathbb{N}$ with intensity measure $M:=\kappa \otimes \#$ where \# stands for the counting measure on $\mathbb{N}$. This means that for a measurable set $A \subseteq \mathcal{P} \times \mathbb{N}$ with $M(A)<\infty$, the counting process

$$
\left.N^{A}(t)=\operatorname{Card}(s \in[0, t]:(\Delta(s), k(s)) \in A), t \geq 0\right)
$$

is a Poisson process with intensity $M(A)$, and to disjoint sets correspond independent processes.

Then one can construct a unique $\mathcal{P}$-valued process $\Pi_{\kappa}=\left(\Pi_{\kappa}(t), t \geq 0\right)$ started from the trivial partition, with càdlàg sample paths, such that $\Pi_{\kappa}$ only jumps at time $t$ at which $K$ has an atom $(\Delta(t), k(t))$, and in that case $\Pi_{\kappa}(t)$ is the partition whose blocks are the $B_{i}(t-)$ (the blocks of $\Pi_{\kappa}(t-)$ ) except for $B_{k(t)}(t-)$ which is replaced by the partition of $B_{k(t)}(t-)$ induced by $\Delta(t)$ (that is $\Delta(t) \cap B_{k(t)}(t-)$ ).

$\Pi_{\kappa}$ is a homogeneous $\mathcal{P}$-fragmentation with characteristic measure $\kappa$. Conversely, any homogeneous $\mathcal{P}$-valued fragmentation $\Pi$ has the same law as $\Pi_{\kappa}$ for some unique exchangeable measure $\kappa$.

As a consequence of Kingman's representation of exchangeable partitions [15], every exchangeable partition measure can be decomposed into the sum of a dislocation measure and an erosion measure:

- $\delta_{\pi}$ stands for the Dirac point mass at $\pi \in \mathcal{P}$, for all $n \in \mathbb{N}$ let $\epsilon_{n}$ be the partition of $\mathbb{N}$ with only two non-void blocks: $\{n\}$ and $\mathbb{N} \backslash\{n\}$, then for every $c \geq 0$, the measure

$$
\mu_{c}=c \sum_{n=1}^{\infty} \delta_{\epsilon_{n}}
$$

is an exchangeable measure. The $\mu_{c}$ 's are called erosion measures.

- The dislocation measures are constructed from so-called Lévy measures on $\mathcal{S}^{\downarrow}$. We call a measure $\nu$ on $\mathcal{S} \downarrow$ a Lévy measure if $\nu$ does not charge $(1,0,0, \ldots)$ and verifies the integral condition

$$
\int_{\mathcal{S} \downarrow}\left(1-s_{1}\right) \nu(\mathrm{d} s)<\infty
$$

where $s=\left(s_{1}, s_{2}, \ldots\right)$ denotes a generic sequence in $\mathcal{S}^{\downarrow}$. The mixture of paintbox processes

$$
\mu_{\nu}(.)=\int_{\mathcal{S} \downarrow} \mu_{s}(.) \nu(\mathrm{d} s)
$$

is a measure on $\mathcal{P}$, called the dislocation measure directed by $\nu$.

Then for any $\kappa$ exchangeable partition measure there exists a unique $c \geq 0$ and a unique Lévy measure $\nu$ such that $\kappa=\mu_{c}+\mu_{\nu}$.

Thus the law of a homogeneous $\mathcal{P}$-fragmentation is completely characterized by the pair $(\nu, c)$. Using Proposition 2.6, we conclude that:

Corollary 3.1. There is a bijective correspondence between the laws of homogeneous ranked fragmentations and the pairs $(\nu, c)$ where $\nu$ is a Lévy measure on $\mathcal{S}^{\downarrow}$ and $c \geq 0$.

A ranked fragmentation is thus completely characterized (in terms of distribution) by the pair $(\nu, c)$ associated to its law. 
We would like to transfer the Poisson point process construction of $\mathcal{P}$-fragmentations to $\mathcal{S} \downarrow$-fragmentations. The main difficulty in doing so comes from the lack of a genealogy structure in this new setting ${ }^{4}$.

To illustrate this, let $K=(\Delta(t), k(t))$ a PPP with measure intensity $\mu_{\nu} \otimes \#$ and $\Pi$ the corresponding $\mathcal{P}$ fragmentation (hence with no erosion), and suppose that at time $t$ there is an atom $(\Delta, k)$ : the $k$-th block of $\Pi(t-)$ (i.e. its least element is $k$ ) fragments, or otherwise said $\Lambda\left(B_{k}(t-)\right)>\Lambda\left(B_{k}(t)\right)>0$. Then it is clear that at time $t$ there is also a dislocation in the associated ranked fragmentation $\lambda=\Lambda(\Pi)$. The label of the mass of $\lambda(t-)$ that fragments, noted $\Phi(t-, k)$, is an integer that depends on $\Pi(t-)$ and $k$ and can informally be seen as the rank of the size of the $k$-th block of $\Pi(t-)$. In the same way that $\Pi$ is constructed from $K$, one might hope that $\Lambda(\Pi)$ is constructed from $(\Lambda(\Delta(t)), \Phi(t-, k(t)))$ but we will still have to show that this last point process is a Poisson point process with the right intensity, then that the jump-times of $\lambda$ are exactly the atom times of $K$ and finally that $\lambda$ is a pure-jump process (in a sense to be defined).

But first we show how to get rid of erosion.

\subsection{Erosion in homogeneous ranked fragmentations}

Let us first examine the trivial case when the fragmentation is pure erosion. It is then intuitively clear that the homogeneity in time and space entails that the ranked fragmentation $\lambda(t)$ with values in $S^{\downarrow}$ with characteristics $(0, c)$ (where the 0 means that the measure $\nu$ is trivial with mass 0 ) is given by

$$
\lambda(t)=\left(\mathrm{e}^{-c t}, 0,0, \ldots\right)
$$

To demonstrate this define

$$
k=\mu_{c}
$$

with $c>0$, and let $\Pi$ be the $\mathcal{P}$-fragmentation associated to the P.P.P. $K=(\Delta(t), k(t))_{t \geq 0}$ with intensity $\mu_{c} \otimes \#$ and values in $\mathcal{P} \times \mathbb{N}$. $\Pi$ can be thought of as an isolation process, indeed at each jump time of $K$, say $t$, some point of $\mathbb{N}$, say $n$, is designated, (i.e. $\Delta(t)=\delta_{\epsilon_{n}}$ ). If the block containing $n, \beta(n, t)$, is not reduced to the singleton $\{n\}$, then it is fragmented into $\{n\}$ and $\beta(n, t) \backslash\{n\}$, " $n$ is isolated from its block", or else nothing happens. Hence, at all time there is only one block which is not a singleton, by an argument that will be established in Theorem 3.3, we can always suppose that this block also contains 1 . If we consider the restriction of $\Pi$ to $\{1,2, \ldots, n\}$, denoted by $\Pi^{(n)}$, then $\Pi^{(n)}$ only jumps at atom-times of $K$ for which $k_{t}=1$ and $\Delta_{t} \in\left\{\delta_{\epsilon_{1}}, \delta_{\epsilon_{2}}, \ldots, \delta_{\epsilon_{n}}\right\}$. The restriction of the Poisson process to this set is a Poisson process with intensity of finite mass and thus have discrete jump-times. The processes of the times of exclusion of each point are independent of one another. By standard calculation on Poisson processes the probability that a given point have been excluded at time $t$ is $\exp (-t c)$, thus the law of the number of points excluded at time $t$ is a Bernoulli with parameter $\left(\mathrm{e}^{-c t}\right)$. By the law of the large numbers, at time $t$, the asymptotic frequency of the only block not reduced to a singleton is $\left(\mathrm{e}^{-c t}\right)$ almost surely. So a.s. for every $t \in \mathbb{Q}$

$$
\Lambda\left(\Pi_{t}\right)=\left(\left(\mathrm{e}^{-c t}\right), 0,0, \ldots\right)
$$

and as $\lambda_{1}(t)$ is monotone decreasing the relation holds almost surely for all $t$. This result is the key for the following:

Proposition 3.2. If $\tilde{\lambda}$ is a homogeneous $(\nu, 0)$ ranked fragmentation, then $\lambda=\left(\mathrm{e}^{-c t} \tilde{\lambda}(t), t \geq 0\right)$ is a homogeneous $(\nu, c)$ ranked fragmentation.

Proof. Let $\tilde{\Pi}$ and $\Pi$ be some homogeneous partition fragmentations with characteristics $(\nu, 0)$ and $(\nu, c)$ respectively. Then call $\tilde{\lambda}$ the process of the ordered asymptotic frequencies of $\tilde{\Pi}$ and $\lambda$ those of $\Pi$. Suppose $\Pi$ is constructed from the Poisson point process $K=(\Delta(t), k(t), t \geq 0)$ with characteristic measure $\mu_{\nu}+\mu_{c}$. Let

\footnotetext{
${ }^{4}$ Of course the branching character of ranked fragmentation that comes from the fragmentation property basically enforces some kind of genealogical structure. By lack of genealogy we only mean that, given $\lambda(t)$ and $\lambda(t+s)$ it is not always possible to determine for a given fragment $\lambda_{i}(t+s)$ from which fragment of $\lambda(t)$ it detached, whereas this is always possible for $\mathcal{P}$-fragmentations.
} 
$K_{1}=(\Delta(t), k(t), t \geq 0)$ the Poisson point process with characteristic measure $\mu_{\nu} \otimes \#$ and $K_{2}=(\Delta(t), t \geq 0)$ the Poisson point process with characteristic measure $\mu_{c}$.

Thus $\Pi$ appears as (i.e. is equal in law to) the intersection of $\Pi_{1}$ (constructed from $K_{1}$ ) and a pure erosion process $\Pi_{2}$ (constructed from $K_{2}$ ), i.e. $\Pi=\Pi_{1}(.) \cap \Pi_{2}($.$) defined by the equivalence relation$

$$
\forall i, j \in \mathbb{N}:\left(i \stackrel{\Pi_{1}(.) \cap \Pi_{2}(.)}{\sim} j\right) \Leftrightarrow\left(\left(i \stackrel{\Pi_{1}(\cdot)}{\sim} j\right) \text { and }\left(i \stackrel{\Pi_{2}(.)}{\sim} j\right)\right)
$$

Given a random exchangeable subset of $\mathbb{N}$, say $A$, independent of $\left(\Pi_{2}(t)\right)_{t \geq 0}$, with random asymptotic frequency $l$, the asymptotic frequency of the subset of $A$ defined as the points that have not been excluded up to time $t$ is $l \mathrm{e}^{-c t}$ a.s. for all $t$.

Therefore $\Lambda(\Pi(t)) \stackrel{\mathcal{L}}{=} \mathrm{e}^{-c t} \Lambda\left(\Pi_{1}(t)\right)$. As we can always suppose that a ranked fragmentation is the associated ranked fragmentation of some partition fragmentation the result is proven.

Thus it suffices to know how to construct a homogeneous ranked fragmentation without erosion from a PPP to know how to construct any homogeneous ranked fragmentation.

\subsection{Construction of homogeneous ranked fragmentations with no erosion}

Let $\lambda$ be an $\mathcal{S}^{\downarrow}$-fragmentation, with characteristics $(\nu, c)$, then for every $k \in \mathbb{N}$ the process $\lambda_{1}(t)+\cdots+\lambda_{k}(t)$ is monotone decreasing. $\lambda$ is said to be a pure jump process if for any $k, \lambda_{1}(t)+\cdots+\lambda_{k}(t)$ is a pure jump process.

In the following we shall focus on the case where for each fixed $t$ there is a infinite number of fragments of strictly positive size almost surely. A necessary and sufficient condition for this is

$$
\nu\left(s \in \mathcal{S}^{\downarrow}: s_{2}>0\right)=\infty .
$$

Indeed, fix $t>0$ and suppose that $\lambda_{1}(t)>0$. Then, for any $\epsilon>0$, during the time interval $[t-\epsilon, t], \lambda_{1}$ has been affected by an infinite number of dislocations such that at least one small fragment detached from the main one, thus an infinite number of fragments have been created, and the life-time of those variables form a sequence of independent identically distributed random variables, thus with probability one an infinite number of them have survived at time $t$. The same line of arguments also shows that $\inf \left\{t \geq 0: \lambda_{1}(t)=0\right\}=\infty$ almost surely.

If

$$
\nu\left(s \in \mathcal{S}^{\downarrow}: s_{2}>0\right)<\infty,
$$

the dislocation times for a tagged fragment are almost surely discrete (in particular almost surely there is a strictly positive random time before which there is only one fragment). Nevertheless, it is possible that at a positive time an infinite number of nonzero components are present with positive probability (a single dislocation could generate a infinite number of fragments).

Although the following theorem is still true for any Lévy measure $\nu$, making this hypothesis enables us to focus on the most interesting case and to avoid some technical difficulties.

Theorem 3.3. Let $\lambda$ be a homogeneous $\mathcal{S} \downarrow$-fragmentation with no erosion $(c=0)$ and Lévy measure $\nu$ as above (i.e. $\nu\left(\left\{s: s_{2}>0\right\}=\infty\right)$. Then

1. $\lambda$ is a pure jump process;

2. there exists a PPP $K=(S(t), k(t))_{t \geq 0}$ with values in $\mathcal{S}^{\downarrow} \times \mathbb{N}$ and intensity measure $\nu \otimes \#$, such that the jumps of $\lambda$ correspond to the atoms of $K$. More precisely, $\lambda$ only jumps at times at which $(S(t), k(t))$ has an atom, and at such a time $\lambda(t)$ is obtained from $\lambda(t-)$ by dislocating the $k(t)$-th component of $\lambda(t-)$ by $S(t)$ (i.e. replacing $\lambda_{k(t)}(t-)$ by the sequence $\lambda_{k(t)}(t-) S(t)$ ) and reordering the new sequence of fragments. Conversely if $(S(t), k(t))$ is an atom then $\lambda$ has a jump at $t$, i.e. $\lambda_{i}$ jumps at $t$ for some $i$.

Although this result is intuitive in regard to the equivalence between $\mathcal{P}$ and $\mathcal{S} \downarrow$ fragmentation, it requires some technical work. 
We give ourselves a homogeneous $(\nu, 0) \mathcal{S}^{\downarrow}$-fragmentation $\lambda$ with $\nu$ satisfying $\nu\left(s_{2}>0\right)=\infty$. There is no loss of generality in supposing that $\lambda$ is constructed as follows: call $K=((\Delta(t), k(t)))_{t>0}$ a PPP with intensity measure $\mu_{\nu} \otimes \#$ with values in $\mathcal{P} \times \mathbb{N}$. Let $\Pi$ be the homogeneous $(\nu, 0) \mathcal{P}$-fragmentation constructed from $K$, then define

$$
\lambda=\Lambda(\Pi) .
$$

Call $\mathcal{F}_{t}=\sigma\left\{\Pi_{s}, s \leq t\right\}$ the natural filtration of the $\mathcal{P}$-fragmentation $\Pi$.

Then at any time $t$, call $\phi(t,)=.\phi_{t}($.$) the random, \mathcal{F}_{t}$ measurable function from $\mathbb{N} \rightarrow \mathbb{N} \cup \infty$ (where $\infty$ serves as a cemetery point) defined as

- if $\left|B_{k}(t)\right|>0$ then $\phi(t, k)$ is the rank of the asymptotic frequency of $B_{k}(t)$ (it is well defined because the number of blocks of greater asymptotic frequencies is always finite with an upper bound of $\left|B_{k}(t)\right|^{-1}$, and in case two blocks have the same asymptotic frequency, they are ranked as their least element);

- if $\left|B_{k}(t)\right|=0$ (with the convention $|\varnothing|=0$ ) then $\phi(t, k)=\infty$

We also define $\tilde{k}(t)=\phi(t-, k(t))$. Note that under our hypothesis that there is always an infinite number of fragments $\forall t \geq 0, \mathbb{N} \subset\{\phi(t, k), k \in \mathbb{N}\}$.

We will first prove that the point process image of $K$, denoted $\tilde{K}$, whose atoms are the points of $(\Lambda(\Delta(t)), \tilde{k}(t))_{t \geq 0}$ such that $\tilde{k}(t) \in \mathbb{N}$, is a Poisson point process with intensity measure $\nu \otimes \#$. Then we will show that this is also the process of the jumps of $\Lambda(\Pi)$ and this last process is a pure jump process so it can wholly be recovered from $(\Lambda(\Delta(t)), \tilde{k}(t))_{t \geq 0}$. This will complete the proof of Theorem 3.3.

Lemma 3.4. The point process $\tilde{K}(t)$ derived from $(\Lambda(\Delta(t)), \tilde{k}(t))_{t \geq 0}$ by only keeping the atoms such that $\tilde{k}(t) \neq$ $\infty$ is a Poisson point process with intensity measure $\nu \otimes \#$.

Proof. Let $A$ be a subset of $\mathcal{S}^{\downarrow}$ such that $\nu(A)<\infty$. For $i=1, \ldots$ let

$$
N_{A}^{(i)}(t)=\#\{u \leq t: \Lambda(\Delta(u)) \in A, k(u)=i\}
$$

Then set

$$
N_{A}(t)=\#\{u \leq t: \Lambda(\Delta(u)) \in A, \tilde{k}(u)=1\}
$$

$N_{A}(t)$ is increasing, right-continuous with left-limits with jumps of size 1 (the $N_{A}^{(i)}(t)$ being independent Poisson processes they do not jump at the same time almost surely). By definition we have

$$
\mathrm{d} N_{A}(t)=\sum_{i=1}^{\infty} \mathbf{1}_{\{\phi(t-, i)=1\}} \mathrm{d} N_{A}^{(i)}(t)
$$

Define

$$
\mathrm{d} \tilde{N}_{A}^{(i)}(t)=\mathbf{1}_{\{\phi(t-, i)=1\}} \mathrm{d} N_{A}^{(i)}(t)
$$

It is clear that $\mathbf{1}_{\{\phi(t-, i)=1\}}$ is adapted and left-continuous in $\left(\mathcal{F}_{t}\right)$ and hence predictable. The $N_{A}^{(i)}($.$) are i.i.d.$ Poisson processes with intensity $\nu(A)$ in $\left(\mathcal{F}_{t}\right)$. Thus, for each $i$ the process

$$
M_{A}^{(i)}(t)=\tilde{N}_{A}^{(i)}(t)-\nu(A) \int_{0}^{t} \mathbf{1}_{\{\phi(u-, i)=1\}} \mathrm{d} u=\int_{0}^{t} \mathbf{1}_{\{\phi(u-, i)=1\}} \mathrm{d}\left(N_{A}^{(i)}(u)-\nu(A) u\right)
$$

is a square integrable martingale. Then define

$$
M_{A}(t)=\sum_{i=1}^{\infty} \int_{0}^{t} \mathbf{1}_{\{\phi(u-, i)=1\}} \mathrm{d}\left(N_{A}^{(i)}(u)-\nu(A) u\right) .
$$


Define $f_{i}(t)=\mathbf{1}_{\{\phi(t-, i)=1\}}$, then, for all $i \neq j, \forall t \geq 0, f_{i}(t) f_{j}(t)=0$, and $\forall t, \sum_{i=1}^{\infty} f_{i}(t)=1$.

As the $N_{A}^{(i)}(t)$ are independent Poisson processes they do not jump simultaneously and so the martingales $M_{A}^{(i)}(t)$ do not either. They are thus orthogonal (see for example Chap. 8, Th. 43-D in [12] for a proof). Moreover the oblique bracket of $M$ is

$$
\left\langle M_{A}\right\rangle(t)=\sum_{i=1}^{\infty}\left\langle\int_{0}^{t} f_{i}(u) \mathrm{d}\left(N_{A}^{(i)}(u)-\nu(A) u\right)\right\rangle=\nu(A) t .
$$

So $M_{A}$ is a $L_{2}$ martingale.

So we have demonstrated that $N_{A}(t)$ is increasing, right-continuous, left limited with jumps of size 1 with compensator $\nu(A) t$. Using classical results (see for instance Chap. 2.6 in [14], Th. 6.2) we conclude that $N_{A}(t)$ is a Poisson process with intensity $\nu(A)$. Now take $B \in \mathcal{S}^{\downarrow}$ such that $A \cap B=\varnothing$, we can use the same construction as above replacing $A$ with $B$ and the fact that $N_{A}^{(i)}(t)$ and $N_{B}^{(i)}(t)$ are independent Poisson processes in the same filtration to see that

$$
N_{1}(t)=\{\Lambda(\Delta(u)): u \leq t, \tilde{k}(u)=1\}
$$

is a P.P.P. with intensity measure $\nu$. The same arguments yield that

$$
N_{2}(t)=\{\Lambda(\Delta(u)): u \leq t, \tilde{k}(u)=2\}
$$

is also a P.P.P. with intensity measure $\nu$. It is clear that $N_{1}$ and $N_{2}$ have no jumps in common because the $N_{A}^{(i)}(t)$ 's do not, so they are independent. By iteration this shows that $(\Lambda(\Delta(t)), \tilde{k}(t))$ is a P.P.P. with intensity measure $\nu \otimes \#$.

Introduce the blocks $\Pi=(\Pi(t), t \geq 0)=\left(\left(B_{1}(t), B_{2}(t), \ldots\right), t \geq 0\right)$ where $\Pi$ is the $(\nu, 0) \mathcal{P}$-fragmentation constructed from $K$, and define $\lambda=\Lambda(\Pi)=\left(\lambda_{1}(t), \lambda_{2}(t), \ldots\right)$ the ordered vector of asymptotic frequencies. In the case considered here $\Pi$ is nice so almost surely for all $t\left|B_{i}(t)\right|$ exists for all $i \in \mathbb{N}$. Recall that $\phi(t, k)$ is the rank of the asymptotic frequency $\left|B_{k}(t)\right|$ at time $t$.

We now need to show that $\lambda$ is a pure jump process in the sense that for each $k$ the decreasing process $\lambda_{1}+\cdots+\lambda_{k}$ is pure jump and that all its jumps are indeed images of some atoms of $K$ ( $\Lambda$ being not continuous it is not a priori evident).

In [5] it is shown that $\left|B_{1}(t)\right|$, the asymptotic frequency of the block that contains $\{1\}$, is the inverse of the exponential of a subordinator with 0-drift, and so it is a pure-jump process. By the Markov and homogeneity property this implies that for all $i>1$ the process $\left|B_{i}(t)\right|$, the asymptotic frequency of the block whose least element is $i$, is càdlàg, started at 0 , such that at $\tau_{i}=\inf \left\{t \geq 0:\left|B_{i}(t)\right|>0\right\}$ we have that conditionally on $\tau_{i}<\infty,\left|B_{i}\left(\tau_{i}\right)\right|>0$ (i.e. it leaves 0 with a jump), and after $\tau_{i}$ the process $\frac{\left|B_{i}\left(t-\tau_{i}\right)\right|}{\left|B_{i}\left(\tau_{i}\right)\right|}$ is the inverse of the exponential of a subordinator with no drift, in particular it is a pure jump process. Furthermore it is clear by construction that all the jumps of $B_{i}($.$) correspond to some atom of \tilde{K}$.

For each $t$ define $\psi_{t}($.$) the function from \mathbb{N} \rightarrow \mathbb{N}$ inverse of $\phi(t,$.$) , i.e.$

$$
\psi_{t}(\phi(t, i))=i
$$

(exists because $\phi$ is surjective on $\mathbb{N}$ ).

Lemma 3.5. - for all $k>0, \lambda_{1}(t)+\lambda_{2}(t)+\cdots+\lambda_{k}(t)$ is a pure jump process;

- with probability one, for all $t \geq 0$, if $\lambda$ jumps at $t$, then $\tilde{K}$ has an atom at $t$.

Proof. We will begin by proving the result for $\lambda_{1}$, the size of the largest fragment and then turn our attention to the small ones.

$\lambda_{1}$ is a supremum of a countable family of pure jump processes (the $\left.\left|B_{i}().\right|\right)$. However it is easy to exhibit an example of a supremum of a countable family of pure jump processes that is not a pure jump process. So the 
proof will consist in showing that almost surely on a fixed time interval $\lambda_{1}$ is the supremum of a finite number of pure jump processes.

The key point is to show that given $t>0$ and an arbitrary $\epsilon>0$ we can almost surely choose a $M$ such that

$$
\forall s \leq t \text { and } n>M,\left|B_{n}(s)\right|<\epsilon .
$$

In particular this would mean that we only need to consider a finite number of $B_{i}($.$) to be sure to "catch" a$ fragment whose size is greater than $\epsilon$.

To do this, it is convenient to work with so-called interval fragmentations.

Interval fragmentations are a particular case of the object fragmentations that we presented in the introduction for which the "object" $E$ is simply the interval $[0,1]$ endowed with the Lebesgue measure. More precisely, call $\nu$ the space of the open subsets of $[0,1]$. Elements of $\nu$ admit a unique decomposition in intervals (in the sense that the ordered vector of the lengths is unique). An interval decomposition is a process $F(t)$ with values in $\nu$ such that for any $0 \leq s<t$ one has $F(s) \subseteq F(t)$ i.e. $F(t)$ is finer than $F(s)$.

Take a sequence $\left(u_{i}\right)_{i \in \mathbb{N}}$ of i.i.d. variables uniformly distributed on $[0,1] . F$ is then transformed into a $\mathcal{P}$-process $\Pi$ by the following rule

$$
i \stackrel{\Pi(t)}{\sim} j \Leftrightarrow\left[u_{i}, u_{j}\right] \subseteq F(t)
$$

This last process obviously conserves the refinement property. Moreover, if we define the interval fragmentations to have the scaling and branching properties, then $\Pi$ will be a self-similar $\mathcal{P}$-fragmentation.

We refer to [6] for a precise definition of interval fragmentation and the equivalence between interval fragmentations and partition fragmentations.

There is no loss of generality in supposing that $\Pi$ is constructed from an interval fragmentation $F(t)$ and a sequence $\left(u_{i}\right)_{i \in \mathbb{N}}$ of iid variables uniformly distributed on $[0,1]$.

Denote by $\left(I_{i}(t), i \in \mathbb{N}\right.$ ) the associated ordered lengths of the interval decomposition of $F$ (which are also the associated ordered frequencies of $\Pi(t))$. If $I^{(i)}(t)$ denotes the length of the interval that contains $u_{i}$ in the interval decomposition of $F(t)$, then

$$
I^{(i)}(t)=l_{i}(t)
$$

where $l_{i}(t)=|\beta(i, t)|$ is the asymptotic frequency of the block of $\Pi(t)$ that contains $i$.

Calling $\tau_{n}$ the stopping time $\inf \left\{t>0,\left|B_{n}(t)\right|>0\right\}$ we have that at $\tau_{n}$

$$
\forall i<n n \stackrel{\Pi\left(\tau_{n}\right)}{\sim} i
$$

Thus $u_{n}$ does not belong to any block of $F\left(\tau_{n}\right)$ that contains some $u_{i}$ for any $i<n$, hence the asymptotic frequency of the block of $F\left(\tau_{n}\right)$ that contains $u_{n}$ is bounded from above by $\sup _{i, j \in\{1, \ldots, n\}}\left|u_{i}-u_{j}\right|$ which converges to 0 almost surely when $n \rightarrow \infty$.

Note that

to see that

$$
\sup _{r \in \mathbb{R}^{+}}\left\{\left|B_{n}(r)\right|\right\}=\left|B_{n}\left(\tau_{n}\right)\right|
$$

$$
\lim _{n \rightarrow \infty}\left(\sup _{r>0}\left(\left|B_{n}(r)\right|\right)\right)=0 \text { a.s. }
$$

Now fix $\epsilon>0$ and $n_{0}$ and condition on the events $\left\{\lambda_{1}(T) \geq \epsilon\right\}$, and

$$
\left\{\sup _{n>n_{0}}\left\{\sup _{r>0}\left(\left|B_{n}(r)\right|\right)\right\}\right\}<\epsilon .
$$

Note that the probability of the second event can be taken arbitrarily close to 1 by taking $n_{0}$ sufficiently large.

On this event, for all $r \in[0, T]$ we have that

$$
\lambda_{1}(r)=\max _{i=1, \ldots, n_{0}}\left|B_{i}(r)\right|
$$


Thus $\lambda_{1}($.$) is a pure jump process because all the \left|B_{i}().\right|$ are. Moreover $\lambda_{1}($.$) only jumps at times at which \tilde{K}$ has an atom for the same reason.

Turning our attention to the other fragments, it should be clear that the above discussion also entails that for an arbitrary $\epsilon>0$, for all $s \in[0, t]$ the process $\lambda_{k}(s) \wedge \epsilon$ can almost surely be expressed in terms of a finite number of pure jump processes (the $\left|B_{i}(s)\right|$ for $i=1, \ldots, M$ ) and the $\lambda_{j}(s)$ for $j=1, \ldots, k-1$. Thus inductively, the result is proven. The second point is immediate from this proof.

In conclusion, if we call $\Gamma$ the set of times at which $(\Delta(t), k(t))$ has an atom, then writing $\lambda(t)=\left(\lambda_{1}(t), \lambda_{2}(t), \ldots\right)$ for $\Lambda(\Pi(t))$ :

1. $\lambda($.$) is a pure jump process, càdlàg and starts almost surely from (1,0,0, \ldots)$;

2. if $t \notin \Gamma$,

$$
\lambda(t)=\lambda(t-)
$$

3. if $t$ is a jump-time for $\lambda$, then almost surely $t \in \Gamma$ and $\lambda(t)$ is the reordering of the concatenation of two sequences: $\left(\lambda_{i}(t-)\right)_{\{i \neq k(t)\}}$ and $\lambda_{\phi_{t}(k(t))}(t-) \Lambda(\Delta(t))$.

As $\lambda$ is a pure jump process it is completely defined by this description.

All we have to do now is collect the preceding results: let $K=(\Delta(t), k(t))$ be a Poisson point process with intensity measure $\mu_{\nu} \otimes \#$ and let $\Pi$ the associated $(\nu, 0)$ homogeneous $\mathcal{P}$-fragmentation. Then the Poisson point process $\left(\Lambda(\Delta(t)), \phi_{t-}(k(t))\right)$ and the asymptotic frequency process $\Lambda(\Pi(t))$ have the desired properties, so Theorem 3.3 is proved.

\section{Small time Asymptotic Behavior}

In [3] Aldous and Pitman consider a specific fragmentation associated with the standard additive coalescent. After renormalizing the vector $\left(1-x_{1}(t), x_{2}(t), \ldots\right)$ by $\frac{1}{t^{2}}$ they are able to give its asymptotic distribution when $t \rightarrow 0$ in terms of a stable subordinator of index 1/2. In [6] Bertoin showed that the characteristics of this fragmentation are $c=0, \alpha=1 / 2$ and the Lévy measure, whose support is included in $\left\{s \in \mathcal{S}^{\downarrow}: s_{3}=0\right\}$, is specified by

$$
\nu\left(s_{1} \in \mathrm{d} x\right)=\left(2 \pi x^{3}(1-x)^{3}\right)^{-1 / 2} \mathrm{~d} x, \quad x \in[1 / 2,1[.
$$

In this section we intend to extend this type of results to all ranked fragmentations such that $\alpha \geq 0$. We shall use the Poisson construction of ranked fragmentations we just established to study their asymptotic behavior near 0 . The results we give are very close in spirit to those concerning the asymptotic behavior of subordinators.

A subordinator, say $\xi$, is an increasing Lévy process whose distribution is specified by its Laplace exponent $\Psi$ that is given by the identity

The Lévy-Khintchine formula reads

$$
\mathbb{E}\left(\exp \left\{-q \xi_{t}\right\}\right)=\exp \{-t \Psi(q)\}
$$

$$
\Psi(q)=k+\mathrm{d} q+\int_{] 0, \infty[}\left(1-\mathrm{e}^{-q x}\right) v(\mathrm{~d} x)
$$

where $k \geq 0$ is the so-called killing rate, $d \geq 0$ is the drift coefficient and $v$ a measure on $] 0, \infty\left[\right.$ with $\int(1 \wedge$ $x) v(\mathrm{~d} x)<\infty$, called the Lévy measure of $\xi$.

The asymptotic behavior of these processes is well known, for instance we have results concerning their distribution:

(see Cor. 8.9 in [18]).

$$
\frac{1}{t} P(\xi(t) \in .) \underset{t \rightarrow 0+}{\rightarrow} v(.)
$$

On the other hand, under conditions of regular variation on the tail of $v$, there are also results concerning the sample path behavior of the limsup and the law of the iterated logarithm (see for instance the end of Chap. III in $[4])$. 
Thus to study the asymptotic behavior of a fragmentation we may benefit from the fact that $\left|B_{1}\right|$ (the mass of the block that contains 1) can be described in terms of a subordinator (see [5]).

We focus on the behavior of the largest $\left(\lambda_{1}\right)$ and of the second block $\left(\lambda_{2}\right)$ of a ranked fragmentation even though we have a more general result in the case of so-called binary fragmentations.

Although the study of $\lambda_{1}$ is relatively straightforward, that of $\lambda_{2}$ requires to use some results on recordprocesses theory. Most of those that will be used in this section are well known or are adapted from standard facts that can be found in textbooks, see [8] for instance.

We stress that $\lambda_{2}(t)$ is not monotone. More precisely it decreases when the second largest fragment undergoes a dislocation and can increase when the largest fragment undergoes a dislocation and one of the new fragments created becomes the second largest.

The idea is to use the Poisson construction: near 0 the largest fragment is almost of size 1, thus the second largest fragment is always a "direct son" of the main fragment, and we shall be able to express its law in terms of the distribution of the largest fragment that has detached from the main.

For a general $\mathbb{R}$-valued P.P.P. $K=\left(K_{t}, t \geq 0\right)$ with intensity measure $\mu$ such that $\left.\left.\forall \epsilon>0, \mu(] \epsilon, \infty\right]\right)<\infty$, it is possible to define the associated record process $R(t)$ as follows: at time $t$

$$
R(t)=\max _{s \leq t}\left\{K_{s}\right\}
$$

Let $\lambda$ be a homogeneous $\mathcal{S} \downarrow$ fragmentation with characteristic $(\nu, c)$ constructed from the P.P.P.

$$
K=(S(t), k(t))_{t \geq 0}=\left(\left(s_{1}(t), s_{2}(t), \ldots\right), k(t)\right)_{t \geq 0}
$$

of intensity measure $\nu \otimes \#$. Let $\left(S^{(i)}(t), t \geq 0\right)=\left(s_{j}^{(i)}(t), j=1,2, \ldots ; t \geq 0\right)$ be the P.P.P. with values in $\mathcal{S}^{\downarrow}$ derived from $K$ by keeping the points such that $k(t)=i$ (the second coordinate being always $i$, it is not expressed). So $s_{j}^{(i)}(t)$ is the relative size of the $j$-th block of the dislocation occurring at time $t$ on the $i$-th block. $S^{(i)}$ is a P.P.P. with intensity measure $\nu$. The $\mathbb{R}$-valued point process $\left(s_{j}^{(i)}(t)\right)$ is thus a P.P.P. with intensity

$$
\nu_{j}(\mathrm{~d} x)=\nu\left(\left\{s=\left(s_{1}, s_{2}, \ldots\right) \in \mathcal{S}^{\downarrow}: s_{j} \in \mathrm{d} x\right\}\right) .
$$

Introduce the function

$$
x \rightarrow \bar{\nu}_{2}(x)=\nu\left(s \in \mathcal{S}^{\downarrow}: s_{2} \geq x\right)
$$

from $\left.] 0, \frac{1}{2}\right] \rightarrow \mathbb{R}^{+}$, and denote by $f$ its generalized inverse (i.e. $f(x)=\inf \left\{t: \bar{\nu}_{2}(t) \geq x\right\}$ ).

Note that $\bar{\nu}_{2}($.$) is finite, i.e. for all x>0 \bar{\nu}_{2}(x)<\infty$. To see this, let $b \in[0,1 / 2]$

$$
\int_{\mathcal{S} \downarrow}\left(1-s_{1}\right) \nu(\mathrm{d} s) \geq \int_{\mathcal{S} \downarrow} s_{2} \nu(\mathrm{d} s)=\int_{0}^{1 / 2} x \nu_{2}(\mathrm{~d} x) \geq \int_{b}^{1 / 2} x \nu_{2}(\mathrm{~d} x)>b \bar{\nu}_{2}(b) .
$$

Let $R(t)$ designate the record at time $t$ of the P.P.P. $s_{2}^{(1)}($.$) which is well defined according to the above argument.$

Proposition 4.1. Let

$$
\left.\lambda=(\lambda(t), t \geq 0)=\left(\lambda_{1}(t), \lambda_{2}(t), \ldots\right), t \geq 0\right)
$$

be a homogeneous $\mathcal{S} \downarrow$ fragmentation with characteristic $(\nu, c)$, then

1. there exists a subordinator $\xi$ with drift $c$ and Lévy measure

$$
\left.L(\mathrm{~d} x)=\nu\left(-\log s_{1} \in \mathrm{d} x\right), \quad x \in\right] 0, \infty[
$$

such that

for $t$ small enough a.s.;

$$
\lambda_{1}(t)=\exp (-\xi(t))
$$


2.

$$
\lambda_{2}(t) \sim R(t), \quad t \rightarrow 0+\quad \text { a.s. }
$$

Proof of Proposition 4.1 (1). Suppose first that $c=0$. The general case follows easily.

Let us consider the P.P.P. $\left(s_{1}^{(1)}(t), t \geq 0\right)$. Its atoms are the relative sizes of the largest fragment created each time that $\lambda_{1}$ splits. Its intensity measure is given by $\nu_{1}(\mathrm{~d} x)=\nu\left(s: s_{1} \in \mathrm{d} x\right)$. Introduce the process

$$
\xi(t)=-\log \left(\prod_{u \leq t} s_{1}^{(1)}(u)\right), t \geq 0 .
$$

It is a subordinator with 0 drift and Lévy measure $\nu\left(-\log \left(s_{1}\right) \in \mathrm{d} x\right)$.

Conditionally on $\lambda_{1}(T)>1 / 2$, one has $\lambda_{1}(t)=\mathrm{e}^{-\xi(t)}$ for all $t<T$. Thus $\lambda_{1}(t)=\mathrm{e}^{-\xi(t)}$ for all $t \leq T_{1 / 2}$ where $T_{1 / 2}=\inf \left\{t: \lambda_{1}(t) \leq 1 / 2\right\}$.

For the second point the idea is to describe the asymptotic behavior of $\lambda_{2}$ in terms of the records of $s_{2}^{(1)}$. We begin with the following technical lemma:

Lemma 4.2. Let

$$
\chi_{t}=\left(\prod_{u \in[0, t[} s_{1}^{(1)}(u)\right)\left(\prod_{u \in[0, t[} s_{1}^{(2)}(u)\right),
$$

and suppose $c=0$ (there is no erosion). Then on the event $\left\{\lambda_{1}(t) \geq 1 / 2\right\}$

$$
\chi_{t} R(t) \leq \lambda_{2}(t) \leq R(t)
$$

Proof. As noted earlier, one can suppose that $\lambda($.$) is the asymptotic frequency of some (\nu, 0) \mathcal{P}$-fragmentation $\Pi$, and $K$ is the image of the P.P.P.

$$
(\Delta(.), k(.)) \rightarrow(\Lambda(\Delta(.)), \phi(., k(.)))
$$

with intensity measure $\left(\mu_{\nu} \otimes \#\right)$. At time $t$ we recall the notation $\psi_{t}(1)$ for the least element of the block of greatest asymptotic frequency in $\Pi$, which is well defined.

Define

$$
f_{i}(u)=\mathbf{1}_{\left\{i \stackrel{\Pi(u)}{\sim} \psi_{t}(1)\right\}}
$$

i.e. $f_{i}(u)$ is 1 if at time $u$ the integer $i$ is in the same block that the least element of the largest block at time $t$ and 0 otherwise. $f_{i}(0)=1$ a.s. Thus, almost surely

$$
D_{i}(t)=\inf \left\{u \in[0, t]: f_{i}(u)=0\right\} \wedge t>0 .
$$

Note that as we are on $\left\{\lambda_{1}(t) \geq \frac{1}{2}\right\}, \psi_{t}(1)$ is always in the block of greatest asymptotic frequency of $\Pi(u)$ for any $u \in[0, t]$; so $D_{i}(t)$ is the detachment time of $i$ from the main block (if $i$ is still in the main block, $D_{i}(t)$ is taken equal to $t)$.

Now take $k \geq 2$, and suppose that at time $t$ there are at least $k$ blocks (almost surely the case under our hypothesis) so $\psi_{t}(k)$ (the least element of the block of $k$-th greatest asymptotic frequency in $\Pi$ at time $t$ ) is well defined, almost surely

$$
D_{\psi_{t}(k)}(t)>0
$$

so if we define $\beta(i, u)$ for the block of $\Pi(u)$ that contains $i$ and $D(k, t)=D_{\psi_{t}(k)}(t)$ we have that

$$
\begin{aligned}
\beta\left(\left(\psi_{t}(k)\right), D(k, t-)\right) & =\beta\left(\psi_{D(k, t-)}(1), D(k, t)-\right) \\
\left|\beta\left(\left(\psi_{t}(k)\right), D(k, t)\right)\right| & <\left|\beta\left(\psi_{D(k, t)}(1), D(k, t)\right)\right|
\end{aligned}
$$


(recall that $\beta\left(\psi_{t}(1), t\right)$ is the largest block at time $t$ ). Thus

$$
\lambda_{k}(t) \leq\left|\beta\left(\left(\psi_{t}(k)\right), D(k, t)\right)\right| \leq \lambda_{1}(D(k, t-)) s_{2}^{(1)}(D(k, t)) \leq s_{2}^{(1)}\left(D_{\psi_{t}(k)}(t)\right) .
$$

As obviously

$$
s_{2}^{(1)}\left(D_{\psi_{t}(k)}(t)\right) \leq R(t)
$$

we conclude that

$$
\lambda_{k}(t) \leq R(t)
$$

We now prove the lower-bound part of the lemma.

Let $T(t)=\inf \{u \leq t: R(u)=R(t)\}$ (the "record-time"). Note that for all $u \in[0, t]$ at which $S^{(2)}$ has an atom,

$$
\lambda_{2}(u-) s_{1}^{(2)}(u) \leq \lambda_{2}(u)
$$

This is not an equality because the largest fragment resulting from the dislocation of $\lambda_{2}(u-)$ can be smaller than $\lambda_{3}(u-)$. Then, for all $u \in[0, t]$ not an atom for $S^{(2)}$,

$$
\lambda_{2}(u-) \leq \lambda_{2}(u)
$$

This is due to the fact that $u$ could be an atom for $S^{(1)}$, for which $\lambda_{1}(u-) s_{2}^{(1)}(u)>\lambda_{2}(u-)$. Recalling that we are still conditioning on $\left\{\lambda_{1}(t)>\frac{1}{2}\right\}$ we have, using the fact that $\lambda_{2}$ is a pure jump process, that

$$
\lambda_{2}(T(t))\left(\prod_{u \in[T(t), t[} s_{1}^{(2)}(u)\right) \leq \lambda_{2}(t)
$$

and here again this is not an equality because a reordering might occur.

Then remark

$$
\lambda_{2}(T(t)) \geq R(t)\left(\prod_{u \in[0, T(t)[} s_{1}^{(1)}(u)\right)
$$

In words: at the time of the record $R(t)$, the second fragment resulting from the dislocation of $\lambda_{1}$, is not necessarily $\lambda_{2}$, but in any case it is smaller or equal.

We can combine (4) and (5) to get

$$
\chi_{t} R(t) \leq \lambda_{2}(t)
$$

We can now prove the second part of Proposition 4.1:

Proof of Proposition 4.1 (2). When $c=0$ we now only have to show that $\chi_{t} \underset{t \searrow 0}{\longrightarrow} 1$ almost surely. $\left(\prod_{u \in[0, t[}\left(s_{1}^{(1)}(u)\right)\right)$ 
and $\left(\prod_{u \in[0, t[}\left(s_{1}^{(2)}(u)\right)\right)$ are independent and identically distributed, and on the event $\left(\prod_{u \in[0, t[}\left(s_{1}^{(1)}(u)\right)\right) \geq \frac{1}{2}$ this last quantity is exactly the $\lambda_{1}(t)$ of some $(\nu, 0)$ fragmentation, thus almost surely

$$
\left(\prod_{u \in[0, t[}\left(s_{1}^{(1)}(u)\right)\right) \underset{t \searrow 0}{\longrightarrow} 1
$$

which thus concludes our proof.

Finally in the case of a homogeneous $(\nu, c)$ fragmentation $\lambda$ with $c \geq 0$, the effect of the erosion is just of multiplying the size of each fragment by a factor $\mathrm{e}^{-c t}$. So clearly the upper bound of Lemma 4.2 is still valid, on the other hand we have

$$
\xi(t) \mathrm{e}^{-c t} R(t) \leq \lambda_{2}(t)
$$

and only a slight modification of the proof for the case $c=0$ is needed.

Remark 4.3. In the case where the fragmentations considered are not homogeneous but only self-similar and without erosion, a more technical version of Theorem 3.3 should still hold. One should be able to give a Poisson point process construction of a partition-valued $(\alpha, \nu, 0)$ fragmentation, by using a thinning of the Poisson point process when $\alpha>0$ to decrease the rate of dislocation, and by adding atoms in the case $\alpha<0$ to increase this rate. Most of the technical work developed in Section 3.3 is adaptable to the self-similar setting so one should be able to "pass" this construction from the partition to the $\mathcal{S} \downarrow$ setting.

However, the results in this section on the small-time asymptotic results of homogeneous fragmentations can be extended to self-similar fragmentations of index $\alpha$ only when $\alpha \geq 0$. Lemma 4.2 does not holds when $\alpha<0$, furthermore the convergence of $\chi_{t}$ is not true in this case (informally the reason is that too many dislocations of the second largest fragment accumulate).

When $\alpha>0$ the record like behavior of the small fragments tends to be accentuated because they are "frozen" and do not split a lot once they are born. On the other hand, when $\alpha<0$ small fragments vanish quickly. In [7] Bertoin shows that when $\alpha<-1$, although dislocations occur continuously and that at each splitting an infinite number of fragments are created, at any fixed time $t$ there are almost surely a finite number of fragments of positive mass. Also in that case it holds with probability 1 that $\zeta:=\inf \{t \geq 0: X(t)=(0,0, \ldots)\}<\infty$.

Remark 4.4. In the homogeneous case, if $\bar{\nu}_{2}($.$) is regularly varying with index (-a)$ in $0^{+}, a \geq 0$, classical results of record-processes theory used with Proposition 4.1 show that

$$
\frac{\lambda_{2}(t)}{f\left(\frac{1}{t}\right)} \stackrel{\mathcal{L}}{\rightarrow} L
$$

when $t \searrow 0$ where $L$ is the extreme law with distribution function $\exp \left(-x^{-a}\right)$ and $f$ is the generalized inverse of $x \rightarrow \bar{\nu}_{2}(x)$.

Remark 4.5. Suppose that $\lambda$ is a binary fragmentation, that is $\nu$ has its support in the subset of $\mathcal{S} \downarrow$ defined as $\left\{s \in \mathcal{S}^{\downarrow}, s_{3}=s_{4}=\cdots=0\right\}$ and that $\bar{\nu}_{2}(x)=\nu\left(\left\{s \in \mathcal{S}^{\downarrow}: s_{2} \geq x\right\}\right)$ is regularly varying near 0 with index $-a$. Then using the same ideas as in the above arguments one can show that, if $R_{2}(k, t)$ denotes the $k^{\text {th }}$ record of $s_{2}^{(1)}\left(\right.$.) up to time $t$, then we have the following asymptotic distributions of the renormalized $\lambda_{k}$ for any $k>1$ :

$$
\forall k>1, \text { a.s. } \quad \lambda_{k}(t) \underset{t \searrow^{+}}{\sim} R_{2}(k, t) .
$$

As a consequence

$$
\frac{\lambda_{k}(t)}{f(1 / t)} \stackrel{\mathcal{L}}{\rightarrow} L(k, a)
$$


where $L(k, a)$ is the law with distribution function

$$
F_{k, a}(x)=\left(\sum_{i=0, \ldots, k-1} \mathrm{e}^{-x^{-a}} \frac{\left(x^{-a i}\right)}{i !}\right)
$$

More generally, the convergence in law holds jointly. The limit distribution function for the $N$ largest blocks being given by

$$
f_{N}\left(x_{2}, x_{3}, \ldots x_{N}\right)=\left(\prod_{i=2}^{i=N-1}\left(\exp -x_{i}^{-a}\right)\right) \int_{0}^{x_{N}}\left(\exp -u^{-a}\right) \nu\left(s_{2} \in \mathrm{d} u\right)
$$

for $x_{1}>x_{2}>\cdots>x_{N}$ (see [16] for instance).

I would like to thank two anonymous referees for their very careful reading and insightful comments.

\section{REFERENCES}

[1] D.J. Aldous, Exchangeability and related topics, edited by P.L. Hennequin, Lectures on probability theory and statistics, École d'été de Probabilité de Saint-Flour XIII. Springer, Berlin, Lectures Notes in Math. 1117 (1985).

[2] D.J. Aldous, Deterministic and stochastic models for coalescence (aggregation and coagulation): A review of the mean-field theory for probabilists. Bernoulli 5 (1999) 3-48.

[3] D.J. Aldous and J. Pitman, The standard additive coalescent. Ann. Probab. 26 (1998) 1703-1726.

[4] J. Bertoin, Lévy processes. Cambridge University Press, Cambridge (1996).

[5] J. Bertoin, Homogeneous fragmentation processes. Probab. Theory Related Fields 121 (2001) 301-318.

[6] J. Bertoin, Self-similar fragmentations. Ann. Inst. H. Poincaré (to appear).

[7] J. Bertoin, The asymptotic behaviour of fragmentation processes, Prépublication du Laboratoire de Probabilités et Modèles Aléatoires, Paris 6 et 7. PMA-651 (2001).

[8] N.H. Bingham, C.M. Goldie and J.L. Teugels, Regular variation. Cambridge University Press, Encyclopedia Math. Appl. 27 (1987).

[9] E. Bolthausen and A.S. Sznitman, On Ruelle's probability cascades and an abstract cavity method. Commun. Math. Phys. 197 (1998) $247-276$.

[10] M.D. Brennan and R. Durrett, Splitting intervals. Ann. Probab. 14 (1986) 1024-1036.

[11] M.D. Brennan and R. Durrett, Splitting intervals II. Limit laws for lengths. Probab. Theory Related Fields 75 (1987) 109-127.

[12] C. Dellacherie and P. Meyer, Probabilités et potentiel, Chapitres V à VIII. Hermann, Paris (1980).

[13] S.N. Evans and J. Pitman, Construction of Markovian coalescents. Ann. Inst. H. Poincaré Probab. Statist. 34 (1998) 339-383.

[14] N. Ikeda and S. Watanabe, Stochastic Differential Equations and Diffusion Processes. North-Holland Mathematical Library (1981).

[15] J.F.C. Kingman, The coalescent. Stochastic Process. Appl. 13 (1960) 235-248.

[16] M. Perman, Order statistics for jumps of normalised subordinators. Stochastic Process. Appl. 46 (1993) 267-281.

[17] J. Pitman, Coalescents with multiple collisions. Ann. Probab. 27 (1999) 1870-1902.

[18] K. Sato, Lévy Processes and Infinitly Divisible Distributions. Cambridge University Press, Cambridge, Cambridge Stud. Adv. Math. 68 (1999).

[19] J. Schweinsberg, Coalescents with simultaneous multiple collisions. Electr. J. Probab. 5-12 (2000) 1-50. http://www.math.washington.edu/ ejpecp.ejp5contents.html 